\title{
Distinction Between Difference and Differential Equations of Motion for Synchrotron Motion
}

\author{
J. A. MacLachlan \\ Fermi National Accelerator Laboratory, ${ }^{*}$ Box 500, Batavia IL 60510
}

November 27, 2007

\begin{abstract}
The meticulous development of the differential equations of motion (EOM) and related analysis for synchrotron oscillation has extended over decades [see, for example, references [1], [2], and [3]], but the parallel treatment of the difference equations (turn-by-turn mapping) is less developed. The author has written the difference equations for the mapping[4], that is to say the difference equation EOM, but has not carried the fundamental development much further. A companion paper[5] is an effort to reconcile difference EOM and differential EOM. Practically one finds that both techniques generally give the same numerical solution when applied to precisely the same physical system. However, it is incorrect to assume that the two methods are mathematically equivalent; there are circumstances in which the distinction between them is material. This note develops the commonality of the two formalisms.
\end{abstract}

\section{Introduction}

The complete nonlinear turn-by-turn map (difference equation EOM) was developed by the author using an idealized representation of a sychrotron[4]. In a companion paper[5], an attempt was made to connect the difference EOM to the typical differential treatment. Practically the latter paper succeeded, but there were a couple of points bypassed with plausibility arguements. In current efforts to supplement computer modeling of coupled bunch motion with a peturbative solution of the time-dependent Vlasov equation, the distinction between finite time differences and differentials has been an obstacle that has prompted reconsideration of this topic.

A natural way to develop the two methods in a parallel fashion may be to write the conservation of phase space area, that is, the basis of the Vlasov equation, in difference form. This idea is not developed further, but a direct application to numerical solutions seems reasonable. The agenda here is to obtain differential EOM as close as practicable to the mapping. In the next section it is argued that the difference equations are more fundamental and that discrepency between them and the cognate differential equations results from the assumptions made in deriving the differential equations. The three sections following are devoted to deriving the difference equations. Following that is a section constructing the differential equations and a section on the Hamiltonian and Vlasov equation. Before summarizing in the final section, I indicate how multibunch distributions can be included in the Vlasov equation framework.

\section{The Root of the Distinction}

A fundamental difference between the differetial EOM and a turn-by-turn mapping is the intial assumption in the prior case that the acceleration and the motion around the ring are simultaneous, whereas in the latter case it is assumed that beam spends most of a circulation period in force-free drift and receives one or a few practically instantaneous energy increments per beam turn. Furthermore, parameter changes are assumed to be adiabatic. The

*Work supported by the U.S. Department of Energy under contract No. DE-AC02-76CH03000. 
subject of adiabaticity is a an area of ongoing controversy; in tune with what appears to be the temper of current papers, I exhibit the adiabaticity parameter $\alpha$ and assert that for $|\alpha| \leq .25$ a process may be regarded as adiabatic:

$$
\alpha=\frac{\frac{d}{d t} \ln S_{B}(a)}{\frac{d}{d t} \ln \Omega_{s}(a)},
$$

where $a(t)$ is any parameter, $S_{B}$ is the area of the phase stable region (so-called bucket area), and $\Omega_{s}$ is the small amplitude synchrotron oscillation angular frequency. The number .25 is a bit arbitrary, but it is rather conventional. As long as there is some finite value of $\alpha$ for which nonadiabatic effects are negligible, the following discussion is justified. Because the accelerators considered are synchrotrons not betatrons, the isolated energy kick model is closer to the physical system. However, there are quantities continuous in time that may change fast enough that the adiabatic assumption is not justified. The remedy is to replace the single turn map by iteration of partial turn maps for each of which $\alpha$ is acceptably small. Conceptually this is moving in the direction of the differential EOM by going part way to the $\operatorname{Lim} \Delta t \rightarrow 0$.

Forest[6] has demonstrated that any drift-kick algorithm for solving the differrential EOM is an approximate but fully symplectic integrator. Conversely, the differential equation is a symplectic approximate contiuous representation of some mapping. Therefore, one may use standard analytical techniques developed in the context of differential equations over many years by many individuals. There will be no violation of area conservation in doing so. What is yet missing is what differential Hamiltonian to use. Given the distinction between the initial assumptions, it is not obvious that the standard Hamiltonian is the closest continuous representation of the difference equations. Therefore, I follow the course from the difference equations to the differential Hamiltonian followed in the differential equations paper[5] with improved mathematical detail. The steps are to find closely cognate differential EOM and construct a Hamiltonian from them.

\section{Derivation of the Difference EOM}

In following sections a careful but elementary derivation of the single-particle difference equations (single turn map) for the longitudinal degree of freedom of beam particles in an idealized synchrotron is given. The map is applicable to the simulation of effects of rf parameter programs or collective forces.[7]

A particle circulating in a synchrotron on a trajectory of mean radius $R=C /(2 \pi)$ has average angular velocity

$$
\omega=v / R,
$$

where $v$ is the speed of the particle. Suppose for simplicity that there is a single accelerating gap. If the frequency and amplitude of the $\mathrm{rf}$ is set so that the particle receives whatever energy increment is required to keep $R$ fixed at $R_{s}$ as the magnitude of the average vertical magnetic field $\left\langle B_{y}\right\rangle$ changes, then the particle is called a synchronous particle, the orbit it follows is the synchronous orbit, and its trajectory is a synchronous trajectory. Imagine looking at the output of a beam current pickup with an oscilloscope that has its time base synchronized to the rf system. The signal from the synchronous particle is at a fixed location on the sweep turn after turn. The observed current pulse is the sum of signals from many particles with non-synchronous trajectories. In the conventional operating mode there will be a stable current pulse about the synchronous time over many beam turns; that is, the particle motion is such that trajectories near a synchronous trajectory at one time remain near it for long times. This stability results from so-called "phase focusing" arising from the slope of the rf waveform at the synchronous phase. When the slope has the same sign as $\frac{d \omega}{d E}$, the change in circulation frequency with respect to particle energy, it provides a force directed toward the synchronous particle. For a sinusoidal voltage waveform there are two phases per period at which the amplitude yields the correct energy increment. However, the slope of the waveform has opposite sign at these points. The term "synchronous phase" is reserved for the phase at which the slope of the waveform leads to phase focusing. If the rf system goes through $h$ cycles during the particle circulation period, there are $h$ synchronous trajectories. ${ }^{1}$

\footnotetext{
${ }^{1}$ Depending on $h$ and the momentum aperture there may be also $h-1$ faster moving synchronous particles and/or $h+1$ slower moving ones, but this possibility is not usually realized in practice.
} 
Table 1: Meaning of symbols in equations and text

\begin{tabular}{|c|c|}
\hline Symbol & Meaning \\
\hline$\alpha$ & adiabaticity parameter \\
\hline$S_{B}$ & the area of a phase stable region, the so-called bucket area \\
\hline$p_{\circ}$ & the momentum $[\mathrm{MeV} / \mathrm{c}]$ on the reference trajectory \\
\hline$\tau_{\circ}$ & period of a particle following the reference trajectory [s] \\
\hline$h$ & harmonic number of a synchronous rf system \\
\hline$\phi_{\mathrm{S}}$ & synchronous phase of rf [deg] \\
\hline$\hat{V}$ & maximum amplitude of the rf waveform \\
\hline$\omega_{s}, \omega_{s, n}$ & angular frequency of beam circulation \\
\hline$\Omega_{s}, \Omega_{s, n}$ & angular frequency of synchrotron oscillation \\
\hline$R$ & mean radius of an orbit \\
\hline$R_{s}, R_{s, n}$ & mean radius of the orbit of a synchronous particle \\
\hline$R_{\circ}$ & mean radius of the reference orbit \\
\hline$R_{i, n}$ & mean radius of the orbit of the $i^{\text {th }}$ particle on the $n^{\text {th }}$ turn \\
\hline$C$ & the length of an orbit; $C \equiv 2 \pi R$ \\
\hline$C_{i, n}$ & the length of the orbit of the $i^{\text {th }}$ particle on the $n^{\text {th }}$ turn \\
\hline$v$ & the mean orbital speed \\
\hline$v_{s}, v_{s, n}$ & the mean synchronous speed \\
\hline$\tau_{s}, \tau_{s, n}$ & period of circulation of a synchronous particle \\
\hline$\tau_{i, n}$ & time coordinate of $i^{\text {th }}$ particle on the $n^{\text {th }}$ turn relative to the synchronous time \\
\hline$\tau_{i_{j}, n}$ & time coordinate of $i^{\text {th }}$ particle in $j^{\text {th }}$ bunch on $n^{\text {th }}$ turn relative to the $j^{\text {th }}$ synchronous time \\
\hline
\end{tabular}

Because in the typical circumstance particle trajectories are restricted to the neighborhood of a synchronous trajectory by phase focusing, it is convenient to write the equations for general trajectories in differences of energy and coordinate from a synchronous trajectory so that one has a conventional oscillatory system for the typical case of synchronous acceleration. However, there are other regimes of longitudinal motion of interest like, for example, the perturbed drifting motion in phase displacement acceleration. In this case the synchronous trajectory moves rapidly through the region of longitudinal phasespace occupied by beam particles and may start and end outside the physical aperture. Then the (hypothetical) synchronous particle is quite distinct from the beam particles, and, if the equations of motion are to be useful in this case also, they must not depend on assumptions about small differences between the synchronous trajectory and the trajectories of beam particles. The derivation below retains the idea of synchronous particle and difference coordinates but avoids differential approximations for differences of quantities between the particle trajectory of interest and the synchronous trajectory.

The definition of "synchrotron" generally includes a statement about the constancy of the reference or synchronous trajectory. In what follows this restriction will be loose, allowing small changes in the radius $R_{s}$ of the synchronous orbit independent of the reference orbit of radius $R_{\circ}$ to which the guide field properties are referred. Furthermore, although the derivation is written for a single accelerating gap and a difference step of one circulation period to keep the notation simple, the generalization to multiple gaps and shorter or longer time steps is straightforward. 


\section{Fundamental Equations in (t,E) Coordinates}

Consider the sequence of arrival times of the $i$-th particle at the one rf gap $t_{i, 1}, t_{i, 2}, \ldots, t_{i, n}$, where the end of the $n$-th turn is marked by the $n$-th crossing of the gap. There is a recursive relation

$$
t_{i, n}=t_{i, n-1}+\frac{C_{i, n}}{v_{i, n}}=t_{i, n-1}+\frac{2 \pi R_{i, n}}{\beta_{i, n} c}
$$

where $C_{i, n}$ is the length of the orbit for the $i$-th particle, $R_{i, n}$ is the average radius of its orbit, and $v_{i, n}$ is its average speed. Define finite differences $\Delta R$ and $\Delta \beta$ by

$$
\begin{aligned}
R & =\left(1+\Delta R / R_{s, n}\right) R_{s, n} \\
\beta & =\left(1+\Delta \beta / \beta_{s, n}\right) \beta_{s, n} .
\end{aligned}
$$

Then

$$
t_{i, n}=t_{i, n-1}+\frac{2 \pi R_{s, n}}{\beta_{s, n} c} \cdot \frac{1+\Delta R_{i, n} / R_{s, n}}{1+\Delta \beta_{i, n} / \beta_{s, n}}=t_{i, n-1}+\tau_{s, n} S_{i, n},
$$

where $\tau_{s, n}$ is the circulation period for the synchronous particle and the $S_{i, n}=\omega_{s, n} / \omega_{i, n} \approx 1$ may be called the "slip factor". The slip factor is $1+$ the fraction of a synchronous circulation period which the particle of interest gains or loses with respect to the synchronous particle per turn. At the end of a turn the particle receives an energy increment which depends, of course, on the voltage on the gap at that time:

$$
E_{i, n}=E_{i, n-1}+e V\left(\phi_{n}\right)=E_{i, n-1}+e V\left(h \omega_{s, n} \tau_{s, n} S_{n}\right)
$$

where synchronization of $\phi$ has been taken into account so that the contribution of $2 N \pi h$ from $N$ prior turns has no effect. Also because of synchronization the rf frequency is $h \omega_{s, n}$.

These equations can be read as a mapping $\mathcal{M}$ of a point $\left(t_{i, n-1}, E_{i, n-1}\right)$ to another point $\left(t_{i, n}, E_{i, n}\right)$ in the $(t, E)$ plane. The equations are supposed to represent a conservative process; thus, the mapping should conserve phasespace area. The Jacobian determinant is

$$
J(\mathcal{M})=\frac{\partial\left(t_{i, n}, E_{i, n}\right)}{\partial\left(t_{i, n-1}, E_{i, n-1}\right)}=\left|\begin{array}{ll}
1 & 0 \\
0 & 1
\end{array}\right| \equiv 1 .
$$

Therefore, phasespace area is conserved and the sequence of $t_{i, n}, E_{i, n}$ for different $n$ lie on a curve of constant $H$ for some Hamiltonian $H$. The map $\mathcal{M}$ is in the form of an acceleration-free drift in which the coordinate $t$ changes followed by an impulse in which only the conjugate momentum $E$ changes. Forest[6] points out that any map with these properties will be area preserving. It also represents an approximate, but exactly symplectic, integrator of a related continuous Hamiltonian system. In the present case, however, the related system is considered a continuous approximation to an impulsive system of interest.

So far an idealized system has been treated essentially exactly. However, the system described is not a very exact model of a physical synchrotron. Dôme[8] points out that to describe fully the longitudinal dynamics in a synchrotron one must consider the contribution to the force on the particle from the changing magnetic field, i.e., what is generally called betatron acceleration. This force acts continuously around the the machine. It is, however, small compared to the rf force. If one uses an impulsive approximation for the betatron acceleration one makes a small discretization error in a small term. Furthermore, because changes in the betatron acceleration will be adiabatic, its explicit inclusion is not required. The true synchronous phase will be very slightly different from the synchronous phase calculated from $\dot{p}$ alone, but for typical cases this discrepency is small and irrelevent to the purpose of the calculation. In a case where it is a central consideration, the betaron acceleration contribution can be included as described in ref. [5]. 


\section{Convenient Coordinates}

The difference equations eqs. 6 and 7 are based on comparing the circulation speed of the $i$-th particle to that of the synchronous particle but nonetheless are written in coordinates of integrated circulation time and total energy. It is more natural to consider differences in arrival time and energy between the $i$-th and the synchronous particle:

$$
\begin{aligned}
\tau_{i, n} & =t_{i, n}-t_{s, n} \\
& =t_{1, n}-\sum_{m=1}^{n} \tau_{s, m} \\
& =t_{i, n-1}+\tau_{s, n} S_{i, n}-\tau_{s, n}-\sum_{m=1}^{n-1} \tau_{s, m} \\
& =\tau_{i, n-1}+\left(S_{i, n}-1\right) \tau_{s, n} .
\end{aligned}
$$

The difference $\varepsilon_{i, n}$ between the energy of the $i$-th particle and the synchronous particle satisfies

$$
\begin{aligned}
\varepsilon_{i, n} & =\varepsilon_{i, n-1}+e V\left(h \omega_{s, n}\left[\tau_{i, n}+t_{s, n}\right]\right)-e V\left(\omega_{s, n} t_{s, n}\right) \\
& =\varepsilon_{i, n-1}+e V\left(h \omega_{s, n} \tau_{i, n}+\phi_{s, n}\right)-e V\left(\phi_{s, n}\right),
\end{aligned}
$$

where $\phi_{s, n}$ is the synchronous phase, $i . e$., the phase of the $\mathrm{rf}$ at the arrival time of the synchronous particle. The $\phi$ coordinate is redefined so that the particle at $\phi=0$ receives the synchronizing energy increment. Then,

$$
\varepsilon_{i, n}=\varepsilon_{i, n-1}+e V\left(h \omega_{s, n} \tau_{i, n}\right)-e V(0) .
$$

The steps above have been taken considering a single bunch. When there are multiple bunches or rf systems with different harmonic number running concurrenly, it is convenient to use the $\mathrm{h}=1 \tau$ and designate by $\tau_{i_{j}}$ the $\tau$ relative to the synchronous phase for the $j^{\text {th }}$ bunch. The synchronous phase recurs at the centers

$$
(j-1 / 2) \tau_{s, n} / h, \quad j=1, \ldots, h
$$

of the $h$ bucket intervals.

$$
\tau_{i_{j}, n}=\tau_{i, n}-\left(j-\frac{1}{2}\right) \frac{\tau_{s, n}}{h}
$$

Note from the definition of $S_{n}$, that when a particle is traveling faster than the synchronous particle, $\tau_{i, n}<\tau_{i, n-1}$. Thus particles travel in the $-\tau$ sense. This definition is not entirely conventional, but it is convenient. If it is desired to have the particles circulate in the $+\tau$ sense, the slip factor can be written accordingly. The $\tau$ equation eq. 9 becomes

$$
\tau_{i_{j}, n}-\tau_{i_{j}, n-1}=\left(S_{i_{j}, n}-1\right) \tau_{s, n} .
$$

If $N$ cavities are equally spaced about the ring, have the same voltage, and are phased to have the same phase for the synchronous particle, the effect is represented by mapping each turn with $N$ applications of the map with $1 / N$ of the total voltage and $1 / N$ of the phase slip in each iteration. One can see that in principle it is possible to treat an arbitrary spacing of cavities with differing dispersion between them by representing each of the sections with different coefficients in the difference equations. Simply because the accelerator being modeled has more than one rf gap does not mean, of course, that one should necessarily use multiple maps per turn. The phase slip between gaps is usually very small so that the approximation of one per turn is generally excellent. Because the differential equation, which in some sense corresponds to an infinite number of gaps per turn, generally gives results consistent with the one-turn map, one will map more than once per turn only in unusual circumstances. The small amplitude synchrotron tune is a measure of the phase change per turn; if it exceeds $\mathrm{O}\left(10^{-2}\right)$, a mapping step of one turn is rather coarse, and it could be important to represent more accurately the actual distribution of the cavities.

Just as the circumference can be subdivided by multiple cavities, the drift can be broken into as many segments as the adequate discrete approximation to any continuously distributed force may require. In particular, when one 
evaluates the effect of beam spacecharge in high intensity and/or low energy accelerators, it may not be adequate to approximate the force with a single kick per turn. Koscielniak has found[9] spurious clumping and breakup of the distribution when the integration step for the spacecharge force is too large. However, the drift equation can be applied separately for each of the requisite number of segments with spacecharge kicks interspersed. Techniques for finding the spacecharge forces from the particle distribution are discussed elsewhere, e. g., in refs. [10] and [11].

\section{Construction of Cognate Differential Equations}

The reader should note that the differential equations are to be "constructed", not derived. The following treatment is probably respectable to physicists but may be imperfect mathematically. However, the goal is only to construct an appropriate Hamiltonian to allow use of the tools of analysis for perturbation calculations for checking results of mapping. The grounding of the construction in the difference equations establishes strong parallels between the differential EOM and the map. The difference equations used are the single bunch forms eq. 9 and eq. 11 to keep the notation simple. They may be recast as

$$
\Delta \tau_{n}=\left(S_{n}-1\right) \tau_{s, n}
$$

and

$$
\Delta \varepsilon_{n}=e \hat{V}[f(\phi)-f(0)]
$$

where $f(\phi)$ has amplitude one and is periodic with period $2 \pi / h$. Dividing each side of eqs. 14 and 15 by $\tau_{s, n}$ one has

$$
\frac{\Delta \tau}{\Delta t}=S_{n}-1
$$

and

$$
\frac{\Delta \varepsilon}{\Delta t}=\frac{e \hat{V}[F(\tau)]}{\tau_{s, n}}
$$

where $\Delta t$ has been taken as $\tau_{s, n}$ and $f(\phi)-f(0)$ has been expressed as a function of $\tau . \tau_{s, n}$ is much smaller than $2 \pi / \Omega_{s, n}$ so one can say

$$
\frac{\Delta}{\tau_{s, n}} \sim \frac{d}{d t}
$$

Making the approximation natural in the differential equation context that the rf potential is distributed around the ring and the ansatz that it is the same in the intervals $\frac{\tau_{s, n}}{x} 1 \leq x<\infty$,

$$
\frac{\Delta}{\tau_{s, n} / x} \approx \frac{d}{d t}
$$

The Limit $\Delta t \rightarrow 0$ is the same as Limit $x \rightarrow \infty$. Thus one may (plausibly) write

$$
\dot{\tau}=S-1
$$

and

$$
\dot{\varepsilon}=\frac{e \hat{V}[F(\tau)]}{\tau_{s}} .
$$

Using the lowest order term in the Taylor series for $F$ and $S-1$ one can write the differential equations eqs. 19 and 20 in an intuitively appealing form.

$$
\begin{gathered}
\dot{\tau}=\frac{\eta_{s}}{\beta_{s}^{2} E_{s}} \varepsilon \\
\dot{\varepsilon}=\frac{\omega_{s}}{2 \pi} e \hat{V} \cos \left(h \omega_{s} \tau\right)
\end{gathered}
$$


When $\mathrm{V}$ is sinusoidal,

$$
\dot{\varepsilon}=\frac{h \omega_{s}^{2}}{2 \pi} e \hat{V} \cos \left(\phi_{s}\right) \tau
$$

With a change of variable ${ }^{2}$

$$
\varepsilon=\frac{\omega_{s} \beta^{2} E_{s}}{\eta} \epsilon
$$

and simplication using the small amplitude synchrotron frequency

$$
\Omega_{s}^{2}=-\frac{e h \omega_{s}^{2} \eta \cos \phi_{s}}{2 \pi \beta^{2} E_{s}}
$$

eqns. 21 and 23 become

$$
\dot{\tau}=\Omega_{s} \epsilon
$$

and

$$
\dot{\epsilon}=-\Omega_{s} \tau
$$

\section{The Hamiltonian and Vlasov equation}

The Hamiltonian for the linearized differential equations 26 and 27 is

$$
H_{10}=\frac{\Omega_{s}}{2}\left(\tau^{2}+\epsilon^{2}\right),
$$

where the " 1 " represents the order of approximation and the " 0 " is a place holder for a perturbation index so that the order index will not be confused with the order of some perturbation. The $\epsilon$ energy variable will be retained and $S_{n}-1$ will be modified to

$$
G(\epsilon)=S_{n}(\varepsilon)-1-\frac{\eta}{\beta_{s}^{2} E_{s}} \varepsilon
$$

The first term in $F(\tau)$ also will be written separately so that the full nonlinear differential equations may be represented as

$$
\dot{\tau}=\Omega_{s}[\epsilon+G(\epsilon)]
$$

and

$$
\dot{\epsilon}=-\Omega_{s}[\tau+F(\tau)]
$$

Therefore, to any order in $\tau$ and $\epsilon$

$$
H_{0}=\frac{\Omega_{s}}{2}\left[\tau^{2}+\epsilon^{2}+2\left(\int^{\epsilon} G\left(\epsilon^{\prime}\right) d \epsilon^{\prime}+\int^{\tau} F\left(\tau^{\prime}\right) d, \tau^{\prime}\right)\right] .
$$

Notice that no term in either EOM is a mixed product of powers of $\epsilon$ and $\tau$.

When the EOM are in the canonical Hamiltonian form

$$
\dot{q}=\frac{\partial H}{\partial p} ; \dot{p}=-\frac{\partial H}{\partial q},
$$

the general form of the time-dependent Vlasov equation is

$$
\frac{\partial \Psi}{\partial t}+\frac{\partial H}{\partial p} \frac{\partial \Psi}{\partial q}-\frac{\partial H}{\partial q} \frac{\partial \Psi}{\partial p}
$$

\footnotetext{
${ }^{2}$ suggested by Ruebén Méndez Rodríguez (priv. comm.) in a slightly different context
} 
where $\Psi$ is the phase space density distribution. Usually it is prudent and adequate to look for a peturbative solution.

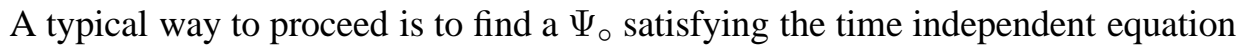

$$
\dot{q} \frac{\partial \Psi_{\circ}}{\partial q}+\dot{p} \frac{\partial \Psi_{\circ}}{\partial p}=0
$$

where the EOM are used to replace the coordinate partials of $H$ by time derivatives. Then the Hamiltonian is separated as $H_{0}+H_{1}$ and the solution is $\Psi=\Psi_{0}+\Psi_{1}$. The perturbation ansatz is that for weak perturbation or small time $\Psi \approx \Psi_{0}$. Stationary $\Psi_{0}$ is guaranteed if $\Psi_{0}=\Psi_{0}\left(H_{0}\right)$. Because generally well localized bunches are wanted, the elliptical, also known as Hoffman-Pedersen[12], distribution is frequently used:

$$
\Psi_{0}(\tau, \epsilon)=\frac{3 N}{2 \pi \hat{\tau}^{3}}\left\{\hat{\tau}^{2}-\epsilon^{2}-\tau^{2}\right\}^{\frac{1}{2}}
$$

where $\hat{\tau}$ is the half bunch length and $N$ is the number of particles in the bunch. For small amplitudes or linearized EOM in general, the bunch is symmetric with extent $\pm \hat{\tau}$ about $\phi_{s}$. However, the definition of the elliptical distribution is not limited to a parabolic potential. The linear charge density is

$$
\lambda(\tau)=\frac{3 N}{4 \hat{\tau}^{3}}\left[\hat{\tau}^{2}-\tau^{2}\right]
$$

\section{Application to Multibunch Distributions}

As suggested in sec. 2, the inclusion of multiple bunches is more a notational challenge than a conceptual one. Basicly the same machinery is used, but the bookkeeping is more involved. For single particle motion, the Hamiltonian for $h$ bunches is a sum of $h$ nearly identical expressions like that for $H_{0}$ in eq. 32. Purely local collective forces like that from comoving images or spacecharge can be included in each single bunch term. When the bunches are coupled by wake fields, the collective effect can be added as an $H_{1}$ which is almost always taken as a perturbation to the uncoupled motion. The perturbative solution consists in essence of solving the single bunch problems that include a potential, which varies from bunch to bunch, calculated from the unperturbed beam current

$$
I_{b, 0}=\lambda(\tau) v_{s}=v_{s} \sum_{j=1}^{h} \lambda_{0}\left(\tau_{j}\right)
$$

where $v_{s}$ is the synchronous velocity and the subscript 0 indicates that $\lambda$ is evaluated from $\Psi_{0}$. Adopting the elliptical distribution for each bunch and assuming them to be identical, only one single bunch distribution is needed to construct $\Psi_{0}$. This perscription is adequate for calculating thresholds and initial growth rates for coupled bunch instability, but a detailed calculation of the unstable motion is most practically approached from the EOM.

\section{Summary}

The EOM for synchrotron oscillation have been considered both as finite difference equations (map) and as ordinary differential equations. It has been shown that the two approaches are very closely related but differ by a fundamental distinction which is ocasionally important in practice. It has been argued that, properly parameterized, a map is more fundamental than the cognate differential equations and is a more faithful model of the physical system. The possibility of an easily negotiated investigation of some finite approach to the limit $\Delta t \rightarrow \infty$ is developed in ref. [13]. In this reference a scaling of the map by a parameter that is the reciprocal of the $x$ appearing eq. 18 is introduced. Because the purpose of that paper is to expand the time step to save computing time, values of $x<1$. are emphasized, but the possibility of $x>1$ is noted. 


\section{Acknowledgement}

This note results from collaborative work on longitudinal coupled bunch motion with Ruebén Méndez. Discussions with him not only suggested to me the need for more clarity in our starting assumptions but also flavored the general approach. I gratefully acknowledge his infuence and specific comments.

\section{References}

[1] J. Moser, Nach. Akad. Wiss. Göttigen IIa No. 6, p87 (1955)

[2] T. Suzuki, Particle Accelerators 12, 237 (1982) Gordon \& Breach publ.

[3] W. T. Weng, "Longitudinal Motion", in Physics of Particle Accelerators, AIP Conf. Proc. 184, pp 243-287, Am. Inst. of Phys., New York (1989)

[4] J. A. MacLachlan, "Difference Equations for Longitudinal Motion in a Synchrotron", FN-529, unpublished Fermilab physics note (December 1989)

[5] J. A. MacLachlan, "Differential Equations for Longitudinal Motion in a Synchrotron", FN-532, unpublished Fermilab physics note (January 1990)

[6] Etienne Forest, "Canonical Integrators as Tracking Codes (or How to Integrate Perturbation Theory with Tracking)", SSC-138 (September 1987), unpublished

[7] J. A. MacLachlan, "Particle Tracking in E - $\phi$ Space as a Design Tool for Cyclic Accelerators", Proc. 1987 IEEE Particle Accelerator Conference held in Washington, D.C., pp1087-1089 (16 - 19 March 1987)

[8] G. Dôme, "Theory of RF Acceleration and RF Noise", in proc. of the CERN Accelerator School Antiprotons for Colliding Beam Facilities, pp215-238, CERN 84-15(20 December 1984)

[9] Shane R. Koscielniak, "The LONG1D Simulation Code”, Proc. of the European Part. Acc. Conf., held in Rome (7-11 June 1988)

[10] J. A. MacLachlan, "Longitudinal Phasespace Tracking with Spacecharge and Wall Coupling Impedance", Fermilab FN-446 (February 1987), unpublished

[11] J. A. MacLachlan, "Fundamentals of Particle Tracking for the Longitudinal Projection of Beam Phasespace in Synchrotrons", Fermilab FN-481, (15 April 1988), unpublished

[12] A. Hofmann and F. Pedersen, "Bunches with Local Elliptic Energy Distribution", Proc. 1979 IEEE Particle Conference, IEEE trans. on Nucl. Sci. Vol. NS-26, No. 3 pp 3526 - 3528 (June 1979)

[13] J. A. MacLachlan and Z. Nazario, "Scaling for faster macroparticle simulation in longitudinal multiparticle dynamics”, PRST-AB 3, 114401 (2000) 